Chronic Obstructive Pulmonary Diseases: Journal of the COPD Foundation

\title{
Journal Club: Phosphodiesterase-4 Inhibitors
}

Ron Balkissoon, MD, MSc, DIH, FRCPC ${ }^{1}$

Abbreviations: phosphodiesterase-4, PDE4; chronic obstructive pulmonary disease, COPD; Global initiative for chronic Obstructive Lung Disease; GOLD; inhaled corticosteroid, ICS; long-acting beta-2 agonist, LABA; long-acting muscarinic antagonist, LAMA; forced expiratory volume in 1 second, FEV $\mathbf{1}$; interleukin, IL; T-helper, TH

Citation: Balkissoon R. Journal Club: Phosphodiesterase-4 inhibitors. Chronic Obstr Pulm Dis (Miami). 2016;3(3):693-697. doi: http:// dx.doi.org/10.15326/jcopdf.3.3.2016.0157

1 Denver, Colorado

\section{Address correspondence to:}

Ron Balkissoon, MD, MSc, DIH, FRCPC

balkissoonr@njhealth.org

\section{Phosphodiesterase-4 Inhibitors: New Insights into Mechanisms of Action and Safety Issues}

Roflumilast is a phosphodiesterase-4 (PDE4) inhibitor that has been approved for use in the United States and several other countries in the last few years for chronic obstructive pulmonary disease (COPD) patients with chronic bronchitis and frequent exacerbations. The Global initiative for chronic Obstructive Lung Disease (GOLD) guidelines recommend it as a treatment option to reduce the risk of exacerbations in patients that typically have 2 or more COPD exacerbations requiring oral steroids or 1 hospitalization in a year. Early studies had shown roflumilast's ability to reduce exacerbations in patients treated with short-acting bronchodilators and long-acting beta agonists but not medications that have been shown to reduce exacerbations such as inhaled corticosteroid/long-acting beta agonist (ICS/ LABA) combinations or the long-acting muscarinic antagonist (LAMA) tiotropium. Last year, Fernando Martinez and colleagues published the paper, "Effect of Roflumilast on Exacerbations in Patients with Severe Chronic Obstructive Pulmonary Disease Uncontrolled by Combination Therapy (REACT): A Multicenter Randomized Controlled Trial," ${ }^{1}$ This study demonstrated that roflumilast was capable of reducing exacerbations and hospitalizations in individuals with COPD who were being treated with an inhaled corticosteroid and long-acting beta-2 agonist combination and were also allowed to be on the LAMA (tiotropium), ( a so called "triple therapy"). Despite these findings there remains some uncertainty for many clinicians as to who are the appropriate patients to consider for roflumilast therapy. In part, this may be related to the fact that, as yet, the exact mechanism by which roflumilast reduces exacerbations has not been fully elucidated. Furthermore, roflumilast comes in a pill form and there have been reports of significant systemic side effects including nausea, diarrhea and weight loss, insomnia, depression and suicide. The recent studies outlined below help to further our understanding of possible mechanisms of action and potential steroid sparing benefits in addition to suggesting there may be potential compromise with regard to the ability to clear lower respiratory pathogens.

\section{Abstract 1 Functional respiratory imaging to assess the interaction between systemic roflumilast and inhaled ICS/LABA/LAMA}

Vos W, Hajian B, De Backer J, et al. Int J Chron Obstruct Pulmon Dis. 2016;11(1):263-271. doi: http://dx.doi. org/10.2147/COPD.S93830

\section{BACKGROUND:}

Patients with COPD show a significant reduction of the lobar hyperinflation at the functional residual capacity level in the patients who improved $>120 \mathrm{~mL}$ in forced expiratory volume in 1 second (FEV1) after 6 months of treatment with roflumilast in addition to inhaled corticosteroids (ICSs)/long-acting beta-2 agonists (LABAs)/long-acting muscarinic antagonists (LAMAs). 


\section{METHODS:}

Functional respiratory imaging was used to quantify lobar hyperinflation, blood vessel density, ventilation, aerosol deposition, and bronchodilation. To investigate the exact mode of action of roflumilast, correlations between lobar and global measures have been tested using a mixed-model approach with nested random factors and Pearson correlation, respectively.

\section{RESULTS:}

The reduction in lobar hyperinflation appears to be associated with a larger blood vessel density in the respective lobes ( $\mathrm{t}=-2.154, P=0.040)$; lobes with a higher percentage of blood vessels reduce more in hyperinflation in the responder group. Subsequently, it can be observed that lobes that reduce in hyperinflation after treatment are better ventilated $(\mathrm{t}=-5.368, P<0.001)$. Functional respiratory imaging (FRI)-based aerosol deposition showed that enhanced ventilation leads to more peripheral particle deposition of ICS/LABA/ LAMA in the better-ventilated areas ( $\mathrm{t}=2.407, P=0.024)$. Finally, the study showed that areas receiving more particles have increased FRI-based bronchodilation $(\mathrm{t}=2.564, P=0.017)$, leading to an increase in $\mathrm{FEV}_{1}$ $(\mathrm{R}=0.348, P=0.029)$.

\section{CONCLUSION:}

The study demonstrated that orally administered roflumilast supports the reduction of regional hyperinflation in areas previously undertreated by inhalation medication. The local reduction in hyperinflation induces a redistribution of ventilation and aerosol deposition, leading to enhanced efficacy of the concomitant ICS/LABA/LAMA therapy. FRI appears to be a sensitive tool to describe the mode of action of novel compounds in chronic obstructive pulmonary disease. Future studies need to confirm the enhanced sensitivity and the potential of FRI parameters to act as surrogates for clinically relevant, but more difficult to measure, end points such as exacerbations.

\section{Comments}

Roflumilast is not considered a bronchodilator and it is not to be used for acute bronchospasm, nonetheless, this study presents data indicating that for a subset of individuals who achieve a greater than $120 \mathrm{ml}$ improvement in forced expiratory volume in 1 second $\left(F E V_{1}\right)$ there is an association with higher blood vessel density and reductions in lobar hyperinflation. In terms of mode of action, this study shows that the use of an oral, systemically-delivered agent has the ability to decrease hyperinflation in areas that previously were difficult to reach by the inhaled route. While the focus of the paper is more on the application of the novel and promising technology of functional respiratory imaging it also provides information with regard to the benefits of orally delivered medications to target small airway disease. It is also instructive with regard to the potential benefit that PDE-4 inhibitors may have for improving the peripheral deposition of inhaled interventions such as ICS/LABA/LAMA therapy.

\section{Abstract 2 Anti-inflammatory effects of the novel inhaled phosphodiesterase type 4 inhibitor CHF6001 on virus- inducible cytokines}

Edwards MR, Facchinetti F, Civelli M, Villetti G, Johnston SL. Pharmacol Res Perspect. 2016;4(1):e00202. doi: http://dx.doi.org/10.1002/prp2.202

Respiratory virus infections precipitate asthma and chronic obstructive pulmonary disease (COPD) exacerbations, with most exacerbations due to rhinovirus infection. Both asthma and COPD exacerbations are not well controlled by steroid therapies, and there is much research interest in finding improved therapies or combinations of therapies for controlling exacerbations. CHF6001 is a new, inhaled highly potent and selective phosphodiesterase type 4 (PDE4) inhibitor. Using in vitro human bronchial epithelial cells (BEAS-2B), we investigated the potential anti-inflammatory effects of CHF6001 on rhinovirus (RV1B)-induced cytokines. Cytokine mRNA was measured by real-time PCR, while protein release was measured by ELISA. CHF6001 was used in a 7-point dose-response curve (1000-0.001 $\mathrm{nmol} / \mathrm{L}$ ) as a 1.5 -h pretreatment prior to infection in comparison with roflumilast. Both roflumilast and CHF6001 reduced RV1B-induced IL-8, IL-29, IP-10, and RANTES mRNA and protein in a concentrationdependent manner. Generally, CHF6001 was 13- to 16 -fold more potent (subnanomolar EC 50 values) than roflumilast at reducing IL-8, IL-29, IP-10, and RANTES $\mathrm{mRNA}$ and protein release, but had similar efficacies. In combination with the steroid fluticasone propionate (1 
nmol/L), CHF6001 had additive effects, significantly reducing RV-induced cytokines when compared with steroid or CHF6001 alone. Combined low-dose steroid and low-dose CHF6001 had a similar efficacy as high-dose steroid or CHF6001 alone, indicating the combination had steroid and PDE4 inhibitor sparing effects. Overall results indicate that PDE4 inhibitors have anti-inflammatory activity against virus-induced inflammatory mediators and that CHF6001 is more potent than roflumilast.

\section{Comments}

As a result of concerns about oral PDE4 inhibitors leading to significant systemic side effects, researchers have been investigating inhaled options. CHF6001 appears to be more potent than roflumilast and has similar efficacy. It is interesting to note the apparent corticosteroid sparing effects of CHF6001 with concomitant treatment with fluticasone propionate given that there have been concerns about the potential increased risk of lower respiratory tract infections with fluticasone propionate. We must determine the risk/benefit ratio between the loss of a systemicallydelivered medication to potentially target small airways versus the potential reduction in systemic side effects from PDE4 inhibition and from corticosteroids.

\section{Abstract 3 The phosphodiesterase 4 inhibitor roflumilast augments the Th17- promoting capability of dendritic cells by enhancing IL-23 production, and impairs their $T$ cell stimulatory activity due to elevated IL-10}

Bros M, Montermann E, Cholaszczyńska A, Reske-Kunz AB. Int Immunopharmacol. 2016;35:174-184. doi: http://dx.doi.org/10.1016/j.intimp.2016.03.025

Phosphodiesterase 4 (PDE4) inhibitors serve to prevent degradation of the intracellular second messenger cAMP, resulting in broad anti-inflammatory effects on different cell types including immune cells. Agents that elevate cAMP levels via activation of adenylate cyclase have been shown to imprint a Th17-promoting capacity in dendritic cells (DCs). Therefore, we studied the potential of therapeutically relevant PDE inhibitors to induce a pronounced Th17-skewing capacity in DCs. Here we show that mouse bone marrow-derived (BM-)
DCs when treated with the PDE4 inhibitor roflumilast (ROF, trade name: Daxas) in the course of stimulation with LPS (ROF-DCs) evoked elevated IL-17 levels in cocultured allogeneic $\mathrm{T}$ cells. In addition, as compared with control settings, levels of IFN- $\gamma$ remained unaltered, while contents of Th2 cytokines (IL-5, IL10) were diminished. ROF enhanced expression of the Th17-promoting factor IL-23 in BM-DCs. In line, neutralizing antibodies specific for IL-23 or IL- 6 when applied to DC/T cell cocultures partially inhibited the IL 17-promoting effect of ROF-DCs. Furthermore, ROFDCs displayed a markedly diminished allogeneic $\mathrm{T}$ cell stimulatory capacity due to enhanced production of IL-10, which was restored upon application of IL-10 specific neutralizing antibody to $\mathrm{DC} / \mathrm{T}$ cell co-cultures. Both the IL-17-inducing and impaired $\mathrm{T}$ cell stimulatory capacity of BM-DCs were mimicked by a specific activator of protein kinase $A$, while stimulation of EPACs (exchange proteins of activated cAMP) did not yield such effects. Taken together, our findings suggest that PDE4 inhibitors aside from their broad overall antiinflammatory effects may enhance the Th17-polarizing capacity in DCs as an unwanted side effect.

\section{Comments}

This study helps to further delineate the antiinflammatory mechanisms of PDE-4 inhibitors with regard to the increased expression of interleukin (IL)-23 in dendritic cells and consequent increased expression of IL-17 in T cells. While T-helper (TH)-17 cells play a key role in neutrophil recruitment, it is important to note that the enhanced IL-10 production does impact the ability of dendritic cells to stimulate $\mathrm{T}$ cell responses. This may potentially impair the ability to eliminate microbes and prevent infections. Given the concerns about inhaled steroids and their association with increased lower respiratory tract infections it behooves us to clarify if the same issues may be of concern with the use of the PDE4 inhibitor class of medications.

\section{Abstract 4 Increases bacterial load and dissemination in a model of pseudomononas aeruginosa airway infection}

Kasetty G, Papareddy P, Bhongir RK, Egesten A. J Pharmacol Exp Ther. 2016; 357(1):66-72. doi: http:// dx.doi.org/10.1124/jpet.115.229641 
Exacerbations present a major clinical problem in many patients suffering from chronic obstructive pulmonary disease (COPD). Roflumilast, an inhibitor of phosphodiesterase 4 , has shown beneficial effects in several clinical trials and is currently widely used to prevent exacerbations in severe COPD. Roflumilast has anti-inflammatory properties that may interfere with potentially important host defense functions, including cytotoxic properties of neutrophils at sites of inflammation. Since chronic bacterial infection is prevalent in severe COPD, Pseudomonas aeruginosa being a major pathogen, we hypothesized that this drug could impair host defense against P. aeruginosa. In this study, mice were pretreated with vehicle alone or roflumilast at doses of $5 \mathrm{mg} / \mathrm{kg}$ or $10 \mathrm{mg} / \mathrm{kg}$, followed by instillation of $\mathrm{P}$. aeruginosa in the airways. Bacterial load and dissemination, as well as inflammatory markers and immune cells, present in the airways were monitored. Roflumilast increased mortality, bacterial load, and dissemination in mice infected with P. aeruginosa. In addition, roflumilast-treated mice had significantly lower numbers of neutrophils in the bronchi, but not in the lung tissue airways, compared with untreated mice. Several proinflammatory cytokines decreased in roflumilast-treated mice but in neither the neutrophilrecruiting chemokine KC nor IL- 6 . These findings show that roflumilast treatment impairs host defense against P. aeruginosa in the airways, which may indicate that patients suffering from chronic bacterial infection of the airways could benefit from withholding of treatment with roflumilast.

\section{Comments}

Studies have shown that roflumilast reduces the risks of exacerbations with or without the use of inhaled corticosteroids or long-acting muscarinic antagonists. While this is an animal study, the findings clearly may have clinical implications with regard to COPD patient selection particularly those showing evidence of chronic infection/dysbiosis (altered microbiome) or who are known to have repeated pseudomonas positive cultures. Human studies are needed that examine the effects of roflumilast in patients with chronic or recurrent infection with Pseudomonas aeruginosa and other common lower respiratory pathogens. 


\section{References}

1. Martinez FJ, Calverley PMA, Goehring U-M, Brose M, Fabbri LM, Rabe KF. Effect of Roflumilast on Exacerbations in Patients with Severe Chronic Obstructive Pulmonary Disease Uncontrolled by Combination Therapy (REACT): A multicenter randomized controlled trial. Lancet. 2015 Mar 7:385(9971):857-66. doi: http:// dx.doi.org/10.1016/S0140-6736(14)62410-7 